Radial and Nonradial Pulsations as Probes of Stellar Physics

ASP Conference Series, Vol. 259, 2002

C. Aerts, T.R. Bedding, \& J. Christensen-Dalsgaard, eds.

\title{
A Comparison of the Anelastic and Subseismic Approximations for Low-Frequency Stellar Oscillations: an Application to Rapidly Rotating Stars
}

\section{B. Dintrans ${ }^{1}$}

Nordic Institute for Theoretical Physics, Copenhagen, Denmark Observatoire Midi-Pyrénées, 14 av. E. Belin, 31400 Toulouse, France

M. Rieutord

Observatoire Midi-Pyrénées, 14 av. E. Belin, 31400 Toulouse, France Institut Universitaire de France

\begin{abstract}
After showing that the anelastic approximation is better than the subseismic one to filter out acoustic waves when studying lowfrequency stellar oscillations, we compute gravito-inertial modes of a typical $\gamma$ Doradus star using this approximation. We show that eigenmodes can be regular or singular, according to the possible focusing towards attractors of the underlying characteristics. Consequences on the oscillations spectrum are then discussed.
\end{abstract}

\section{Introduction}

$\gamma$ Doradus stars are rapidly rotating variables for which the second-order perturbative theory of Ledoux (1951) fails to reproduce observations. Indeed, oscillations and rotation periods are both around one day (Handler \& Krisciunas, 1997) and eigenfrequencies should be non-perturbatively computed.

In this paper, gravito-inertial modes of a typical $\gamma$ Dor star are computed using the anelastic approximation. We first compare the anelastic and subseismic approximations and show that the anelastic one is the best to filter out acoustic waves from the infinite system of differential equations (Sect. 2). Properties of gravito-inertial oscillations are then recalled (Sect. 3) and the part played by the characteristics trajectories is discussed, before conclusions in Sect. 4.

\section{Comparison of the anelastic and subseismic approximations}

These two approximations have been compared in Dintrans \& Rieutord (2001). Both approximations neglect perturbations in the gravitational potential and assume that the Eulerian pressure fluctuations do not contribute to Lagrangian

\footnotetext{
${ }^{1}$ Supported by the European Commission under Marie-Curie grant No. HPMF-CT-1999-00411; email: boris.dintrans@ast.obs-mip.fr
} 
ones. On the contrary, they differ on the form of the equation of mass conservation as

$$
\text { Anelastic: } \vec{\nabla} \cdot\left(\rho_{0} \vec{v}\right)=0, \quad \text { Subseismic: } \quad \vec{\nabla} \cdot \vec{v}=\left(g / c^{2}\right) v_{r}
$$

where $\rho_{0}, g$ and $c^{2}$ respectively denote the equilibrium density, gravity and square of the velocity of sound whereas $\vec{v}$ is the velocity.

Comparisons have been made using g-modes of the homogeneous and $n=3$ polytropes. Analytic expressions for the anelastic and subseismic eigenfrequencies have been found for the homogeneous model whereas all the eigenfrequencies have been computed numerically for the $n=3$ polytrope.

Results are summarized in Table 1 . They show that the anelastic values are between 5 and 20 times more accurate than the corresponding subseismic ones demonstrating that the anelastic approximation is best to filter out acoustic waves when studying low-frequency stellar oscillations.

Table 1. Complete dimensionless eigenfrequencies $\omega^{2}$ for some gmodes of the $n=3$ and homogeneous (in parenthesis) polytropes, with their associated anelastic $\omega_{\text {anel }}^{2}$ and subseismic $\omega_{\text {sub }}^{2}$ counterparts.

\begin{tabular}{lccc}
\hline & $\omega^{2} \times 10^{3}$ & $\omega_{\text {anel }}^{2} \times 10^{3}$ & $\omega_{\text {sub }}^{2} \times 10^{3}$ \\
\hline $\mathrm{g}_{5}$ & $5.76059(-36.1367)$ & $6.03954(-35.2941)$ & $6.27794(-32.6087)$ \\
$\mathrm{g}_{10}$ & $1.98396(-12.2196)$ & $2.02646(-12.1212)$ & $2.08345(-11.5607)$ \\
$\mathrm{g}_{20}$ & $0.59984(-3.65629)$ & $0.60453(-3.64741)$ & $0.61587(-3.55239)$ \\
$\mathrm{g}_{30}$ & $0.28631(-1.73527)$ & $0.28744(-1.73327)$ & $0.29154(-1.70180)$ \\
\hline
\end{tabular}

\section{Application to the long-period oscillations of a $\gamma$ Dor type-star}

The anelastic equations need to be solved in a co-rotating frame; they read (Dintrans \& Rieutord, 2000, hereafter DR2000)

$$
i \sigma \vec{\nabla} \times\left(\vec{v}-\frac{N^{2}}{\sigma^{2}} \vec{v} \cdot \vec{e}_{r}\right)+\vec{\nabla} \times(2 \vec{\Omega} \times \vec{v})=0 \quad \text { and } \quad \vec{\nabla} \cdot\left(\rho_{0} \vec{v}\right)=0
$$

where we assumed a time-dependence of the form $\exp (i \sigma t)$ and $N$ denotes the Brunt-Väisälä frequency. After projecting on vectorial spherical harmonics (see Dintrans et al., 1999 for numerical details), one obtains a generalized eigenvalue problem which is solved using either the QZ algorithm (all the eigenvalues $\sigma$ are computed) or an iterative Arnoldi-Chebyshev solver (only desired eigenvalues $\sigma_{n}$ are computed, with their associated eigenvectors $\vec{v}_{n}$ ).

The perturbative theory has been tested by following a high-order g-mode (period $\sim 0.3 \mathrm{~d}$ ) of a typical $1.5 \mathrm{M}_{\odot} \gamma$ Dor star as rotation was increased. As expected for this kind of long-period mode, perturbative theories rapidly reach their limits since first- and second-order relative errors are comparable to the 

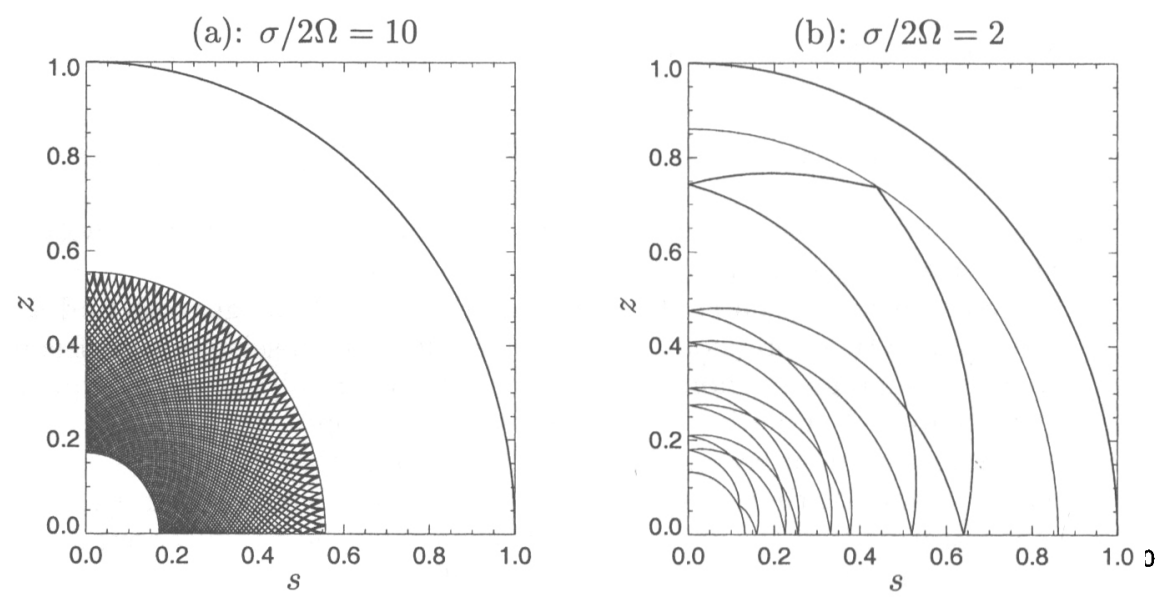

Figure 1. Integration of the characteristics Eq. (2) for a star with a rotation period of one day and two mode frequencies $\sigma$. If $\sigma$ is well above $2 \Omega$ (a), characteristics fill the whole hyperbolic dornain whereas they can be focused along attractors when $\sigma \sim 2 \Omega(\mathrm{b})$. Three hundred reflections have been drawn in both cases but we removed the first two hundred ones in plot (b) to emphasize the final attractor.

observational detection limit for rotation periods of about 8 and 3 days, respectively.

Then, by solving the non-perturbative problem (1) in the rapid rotation regime (rotation down to one day), we found that the part played by the characteristics trajectories become more and more important as the rotation increases. Characteristics, on which energy propagates, obey the following differential equation ( $s$ and $z$ being cylindrical coordinates with $r^{2}=s^{2}+z^{2}$ )

$$
\frac{d z}{d s}=\frac{N^{2} s z \pm r \sqrt{\sigma^{2} N^{2} s^{2}+\left(4 \Omega^{2}-\sigma^{2}\right)\left(\sigma^{2} r^{2}-N^{2} z^{2}\right)}}{\sigma^{2} r^{2}-N^{2} z^{2}} .
$$

Given a mode frequency $\sigma$ and rotation rate $\Omega$, this equation can be integrated in the radiative zone of the star and we found that the resulting web imposes the mode structure depending on the ratio $\sigma / 2 \Omega$ :

- if $\sigma \gg 2 \Omega$, all characteristics trajectories are ergodic (Fig. 1a) and the associated eigenmodes are regular, in the sense that the velocity field is smooth and square-integrable (see Fig. 8a in DR2000).

- if $\sigma \sim 2 \Omega$ (the region of interest), characteristics trajectories almost focus along attractors (Fig. 1b) and the associated eigenmodes are singular, that is, velocity tends to infinity on the attractor (see Fig. 8b in DR2000). Thus, without an efficient diffusion process, these modes cannot exist.

- if $\sigma<2 \Omega$, no global eigenmodes have been found, most likely because of numerical difficulties. 


\section{Conclusion}

First, we showed that the anelastic approximation is better than the subseismic one to filter out acoustic waves from oscillations equations. Second, we applied this result to investigate the low-frequency oscillations of a rapidly rotating $\gamma$ Dor type-star.

We tested first- and second-order perturbative theories by following a longperiod g-mode while increasing rotation. We showed that a non-perturbative approach is necessary for rotation periods below 3 days. Then, a computation of eigenmodes allowed us to reach the one-day rotation region where we found that modes divide into regular and singular ones according to whether the associated characteristics trajectories are ergodic or focused along an attractor. It has important consequences on the oscillation spectrum since frequency subintervals corresponding to attractors are lacking eigenvalues when dissipative effects are neglected.

\section{References}

Dintrans, B., Rieutord, M., \& Valdettaro, L. 1999, J. Fluid Mech., 398, 271

Dintrans, B., \& Rieutord, M. 2000, A\&A, 354, 86 (DR2000)

Dintrans, B., \& Rieutord, M. 2001, MNRAS, 324, 635

Handler, G., \& Krisciunas, K. 1997, Delta Scuti Star Newletter, 11, 3

Ledoux, P. 1951, ApJ, 114, 373

\section{Discussion}

R. Townsend : What advantage does the anelastic approximation give, if you must still use an expansion in spherical harmonics?

B. Dintrans : We always need to expand the angular part of perturbations into spherical harmonics. But adding rotation through Coriolis' force leads to an infinite system of coupled differential equations. The usefulness of the anelastic approximation is precisely to decrease the system size by filtering out acoustic waves, which makes numerics more efficient.

R. Townsend : What is the physical interpretation of the singular modes which you find?

$B$. Dintrans : These modes are due to the focusing of the characteristics, associated with the inviscid problem, towards periodic orbits (attractors); this process is therefore coming from the ill-posedness of the associated mathematical problem. Physically, you may think to a wave packet which, following the characteristics, will bounce on the boundaries. After each cycle, its wavelength is reduced by a factor (which depends on frequency) larger than unity. After an infinite time, the wavelength is zero and the singularity is born. 